\title{
The value of peri-lesional tissue stiffness and stiff rim sign in the differential diagnosis between benign and malignant breast lesions
}

\section{wen-tao Kong}

Drumtower Hospital, Nanjing University https://orcid.org/0000-0002-4313-6958

yin Wang

Drumtower Hospital, Medical College of Nanjing University

wei-jun Zhou

Drumtower Hospital, Medical College of Nanjing University

yi-dang Zhang

Drumtower Hospital, Medical College of Nanjing University

xiao-ming Zhuang

Drumtower Hospital, Medical College of Nanjing University

wen-ping Wang

Zhongshan Hospital, Fudan University

min Wu ( $\nabla$ wuminguyi@163.com )

\section{Research article}

Keywords: Shear wave elastography, Stiff rim sign, Breast, Lesion

Posted Date: August 28th, 2019

DOI: https://doi.org/10.21203/rs.2.10742/v1

License: (c) (i) This work is licensed under a Creative Commons Attribution 4.0 International License. Read Full License 


\section{Abstract}

Background Shear wave elastography (SWE) is an important method in the diagnosis of breast lesions. The purpose of this study was to evaluate the value of tissue stiffness around breast lesion and stiff rim sign for the differentiation of benign and malignant lesions. Methods 192 patients (mean age, $44.6 \pm$ 13.6 years) with 199 breast lesions proven by pathological examination underwent shear wave elastography (SWE). We first observed if there was a stiff rim sign. Then the shell around the breast lesion on SWE was automatically drawn by machine, with width of $1 \mathrm{~mm}, 2 \mathrm{~mm}$ and $3 \mathrm{~mm}$. Elasticity modulus of the lesion and surrounding tissue were recorded, including maximum elasticity (Emax), mean elasticity (Emean), minimum elasticity (Emin) and elasticity ratio (shell/lesion ratio). The optimal thresholds of elasticity modulus were calculated according to receiver operating characteristic (ROC) curve. Results There were 75 malignant lesions and 124 benign lesions. The average Emax, Emean of lesion and shell were significantly higher in the malignant group than in the benign group $(P<0.05)$. The optimal cut-off value of Emax for diagnosing malignant lesion was $101.7 \mathrm{Kpa}$, with a sensitivity of $66.3 \%$ and specificity of $87.9 \%$. The optimal cut-off value of Emean was $29.1 \mathrm{Kpa}$, with a sensitivity of $65.3 \%$ and specificity of $79.8 \%$. The stiff rim sign had a highest diagnostic performance for malignancy than other elastic parameters, with an accuracy of $88.4 \%$. However, measuring peritumoral tissue stiffness can achieve a relatively high sensitivity, whereas specificity was not improved significantly. Conclusion The stiffness of tissue surrounding breast malignancy was significantly higher than benign lesion. Stiff rim sign has the potential to improve the diagnostic performance of breast lesions.

\section{Background}

Breast US elastography is a technique that can be used to assess the stiffness of lesions, which may be helpful for the differential diagnosis. Based on various literatures and evidence-based results, WFUMB Guidelines have recommended additional elastography to conventional US to improve the breast lesion characteristics [1]. Usually, there are two types of elastography methods, strain elastography (SE) or shear wave elastrography (SWE) [2-3].

Compared with SE, SWE has the ability to measure lesion stiffness, so it is regard as a reproducible and quantitative means. Some studies have demonstrated that SWE showed high sensitivity and specificity for the characterization of breast lesions [4-6]. Besides the measurement of intra-lesional stiffness, there is a growing interest of measuring tissue stiffness around the breast lesion. Zhou et al pointed out the strain ratio of surrounding tissue and lesions may be helpful for breast lesions diagnosis [7]. Park et al found malignant breast lesion with a stiff rim were larger in size and associated with more aggressive pathologic subtypes [8]. The surrounding rim of higher stiffness which is characterized by desmoplastic reactions may be due to the infiltration of cancer cells into interstitial tissues or intraductal components [9].

In our study, we used two methods to assess the stiffness of peri-lesional tissue. One was observing whether there was a stiff rim sign or not, the other was making a quantitative measurement of the 
stiffness around lesion (shell). Our aim was to find the value of peri-lesional stiffness in the differential diagnosis between benign and malignant breast lesions.

\section{Methods}

\section{Patients}

From June 2017 to December 2018, 192 patients (mean age $44.6 \pm 13.6$ years; age range, 18-73 years) with breast lesions were included in this study. The enrollment criteria were as follows: $₫$ no previous

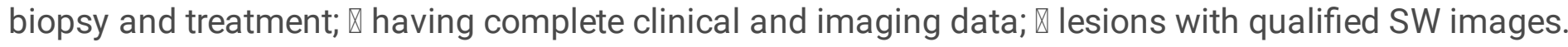
All patients were pathologically confirmed through US-guided core needle biopsy (CNB) or surgery. Informed consent was obtained from all patients and the study was approved by the Ethical Committee of our hospital.

\section{US and shear wave elastography}

Breast US examination was performed using DC-8 diagnostic US system (Mindray Medical International, Shenzhen, China) with a 5-12-MHz linear-array probes. Conventional US was first performed and images were classified according to Breast Imaging and Reporting and Data Systems (BI-RADS) lexicon including shape, margin, orientation, echo pattern, posterior acoustic features and the presence of calcification [10]. US BI-RADS grading results are judged only by ultrasound images, regardless of whether the patient receives mammography or not. After the examination of conventional US, a serious of elastography techniques were then preformed for the lesions by a specialist with at least five years experiences in breast imaging.

We switched ultrasound machine to the SWE mode. Patient was told to hold breath for a few seconds and the probe was placed vertically on the skin surface with slight manual compression. Then the double dynamic mode was used to observe grey-scale image and SWE image. A square region of interest (ROI) was adjusted to include the whole lesion and adjacent breast tissue. Then SWE quality mode and SWE mode were displayed in order. High quality images were presented as uniform green without purple artifact. In the SWE mode, the stiffness of lesions was represented by color map from red (hard) to blue (soft). Two radiologists were blinded to pathological and clinical findings, observing and analyzing the image features. First, the stiff rim sign, defined as red or orange halo around the breast lesion, was observed (fig.1). Then we drew the border of lesion manually. Afterward, the shell just around the breast lesion was drawn, with width of $1 \mathrm{~mm}$ (shell A), $2 \mathrm{~mm}$ (shell B), and $3 \mathrm{~mm}$ (shell C), respectively (fig.2). The maximum (Emax), mean (Emean), minimum (Emin) stiffness value in $\mathrm{kPa}$ of lesion and shell, and the elasticity ratio (shell/lesion ratio) were calculated automatically by the machine. All images were stored in the hard disk for further analysis.

\section{Statistical analysis}


We used SPSS 22.0 (SPSS, Chicago, IL) as the statistical software. SWE values were expressed as mean \pm standard deviation. Quantitative data and Categorical data were analyzed using independent t-test and Chi-square test, respectively. Receiver operating characteristic (ROC) curve was constructed to determine the optimal cut-off values for Emax, Emin, Emean values and shell/lesion ratio. The sensitivity, specificity, accuracy and the area under the curve (AUC) of these parameters were also calculated. $\mathrm{P}<0.05$ was considered statistically significant.

\section{Results}

\section{Pathological diagnosis}

Totally 192 patients with 199 breast lesions were enrolled in our study, including 75 malignant lesions and 124 benign lesions. Of the malignant lesions, 61 were invasive ductal carcinoma (IDC), 8 were ductal carcinoma in situ (DCIS), 4 were mucinous carcinoma, 1 was invasive lobular carcinoma, and 1 was mixed metaplastic carcinoma. Of the benign lesions, 111 were fibroadenoma, 8 were intraductal papilloma, 3 were benign phyllode tomour, and 2 were inflammatory granuloma.

\section{Diagnostic performance of BI-RADS}

The mean diameter of lesions was $1.8 \pm 0.9 \mathrm{~cm}$. There was a significant difference of mean diameter between benign $(1.6 \pm 0.7 \mathrm{~cm})$ and malignant lesions $(2.2 \pm 1.1 \mathrm{~cm})(P<0.05)$. B mode BI-RADS categories were summarized in Table 1. For benign lesions, 58 were category 3, 50 were category 4a, 15 were category $4 \mathrm{~b}$, and 1 was category $4 \mathrm{c}$. Whereas for malignant lesions, the lesion numbers classified as category $4 a, 4 b, 4 c$ and 5 were $9,13,41,12$, respectively. If we set BI-RADS category $4 a$ as the optimal cutoff, the sensitivity, specificity, and accuracy for diagnosing malignant lesions were $88.0 \%, 87.1 \%$, and $75.1 \%$.

\section{Diagnostic performance of SWE}

The Emax and Emean of lesions were significantly higher in the malignant group than in the benign group $(P<0.05)$ (Table 1). According to the ROC analysis, the optimal cut-off was $101.7 \mathrm{Kpa}$ for Emax showing a sensitivity of $65.3 \%$ and specificity of $87.9 \%$. For Emean the optimal cut-off was $29.1 \mathrm{Kpa}$, with a sensitivity of $65.3 \%$ and specificity of $79.8 \%$.

As shown in Table 1, Shell/lesion ratio was higher for malignant lesions than benign lesions, with significant difference $(P<0.05)$. The Emax and Emean of shell $A$, shell $B$ and shell $C$ were also significantly higher in malignancies than in benign lesions $(P<0.05)$. The sensitivity, specificity, and accuracy of the quantitative parameters concerning peri-lesional tissue stiffness for malignancy were shown in Table 2. 
The number of lesions appearing stiff rim sign was 13 in benign group and 66 in malignant lesions, with significant difference $(P<0.05)$. Stiff rim sign had a highest diagnostic performance for malignancy than other elastic modulus, with an accuracy of $88.4 \%$ (Fig.3-5). BI-RADS in combination with stiff rim sign can increase diagnostic sensitivity to $92.0 \%$ and accuracy to $82.3 \%$.

\section{Discussion}

It is well known that elastography is an important method in the differential diagnosis of breast lesions. Different from strain elastography, SWE use high intensity acoustic pulse for tissue compression, causing measurable transverse shear wave. A recent meta-analysis showed the sensitivity, specificity and diagnostic odds ratio of SWE in combination with conventional US were $0.877,0.849$, and 40.164 [11].

The representative technique of SWE is virtual touch tissue imaging quantification (VTIQ) $[12,13]$. Our previous research showed SWVmax value had relative high diagnostic performance with a sensitivity of $74.0 \%$ and specificity of $92.1 \%$, while setting $5.37 \mathrm{~m} / \mathrm{s}$ as the optimal cut-off value. However, one major limitation of VTIQ is the ROI placement may be subjective and show inter-observer variability. In the present study, focal region include all the breast lesion and surrounding tissue. The machine automatically calculates Emax, Emax, Emin of lesion and shell, with no need for selecting ROI. Therefore, it may be a more objective method than VTIQ.

Besides the analysis of intra-tumoral elasticity, some scholars have showed interest in the elasticity of peri-tumoral tissue. Zhou et al [7] may be the first researchers to evaluate the stiffness of peri-lesional tissue. They found there was a significant high positive correlation between the strain ratio of the lesion and the strain ratio of the surrounding tissue in the malignant group $(r=0.740, P<0.001)$. In the study by Xiao et al [14], quantitative elastography features of the lesion rim can significantly improve diagnostic specificity compared with conventional B-mode US alone. The possible reasons of stiff rim sign and high stiffness of surrounding tissue may be due to the microscopic environmental changes in the peri-lesional stroma of malignant breast lesion. As we know, the elasticity is related to the content of collagen fiber [15]. After the increase of angiogenesis and microvessel density, cancer cells infiltrate into the stroma and cause desmoplastic reaction [16]. Another hypothesis is that the stiff peri-pesional tissue may cause attenuation of shear wave, resulting in low shear wave amplitude of internal lesions [8].

In our study, we used two methods to evaluate the surrounding tissue stiffness. One was to quantitatively measure the shell and shell/tumor ratio, the other was to identify whether a stiff rim sign existed or not. The result showed stiff rim sign yielded a satisfactory sensitivity of $88.0 \%$ and specificity of $89.5 \%$, which is higher than the diagnostic performance of lesion elasticity. However, the sensitivity of most quantitative measurement data was high but specificity was relative low. The reason of low specificity may be due to the tissue of shell we chose in our study. The surrounding tissue of breast lesion was glandular tissue, which had higher stiffness than fatty tissue. Therefore, some benign lesions may show high stiffness of shell and shell/tumor ratio. This result was in accordance with the research of Zhou et al [7]. Another possible influence factor was that we cannot draw the outline of the lesion precisely. 
Sometimes the shell may include the breast lesion tissue, thus resulting in relatively low specificity and accuracy. On the contrary, the intra-lesional elasticity had a low sensitivity and relatively high specificity. We found that the sensitivity of Emax and Emean of tumor is $65.3 \%$. This meant about $34.7 \%$ malignant lesions would be missed if we used elasticity of tumour value alone. False negative rate may be due to the size and type of lesion. It has been reported that DCIS, mucinous carcinoma or small breast carcinoma had lower SWV [17].

The stiff rim sign showed a high diagnostic performance compared with other elastography. However, 13 lesions (eleven fibroadenomas, one intraductal papillomas and one mastitis) were considered false positive, whereas nine lesions (six IDCs and 3 DCISs) were considered false negative. We must acknowledge that stiff rim sign is limited in its subjectivity and image quality. Sometimes the red or orange halo around the lesion was incomplete, which was also regard as stiff rim sign. This may reduce the diagnostic sensitivity and specificity.

In our study, we found the sensitivity of BI-RADS was $88 \%$, and its specificity was $87.1 \%$. The high sensitivity and specificity were similar to stiff rim sign, but also higher than quantitative parameters of SWE. The diagnostic performance of SWE was not as effective as we expected. Therefore, we hold the opinion that SWE can only provide additional information of tumor elasticity, and the final diagnosis should rely on the combination of elastography with the BI-RADS classification standard. Our study showed the addition of stiff rim sign to BI-RADS can increase the accuracy (from $75.1 \%$ to $82.3 \%$ ) and sensitivity (from $88.0 \%$ to $92.0 \%$ ). Only three malignancies would be missed if we used combined method. The three false negative lesions were IDCs smaller than $1 \mathrm{~cm}$, which was in line with the result reported by Vinnicombe [18] and Chen [19]. Some malignancies may have benign characteristics when they were small.

There were some limitations in our study. First, the malignancy in this study was mostly invasive ductal carcinoma $(61 / 75,81 \%)$. Therefore, we did not further analyze the elastography of different subtype. Second, we should admit that stiff rim sign was subjective to some extent. Inter-observer variability was not taken into account and could impact our results. Third, it was a single-center study and a large number of cases would be needed.

\section{Conclusion}

The stiffness of tissue surrounding breast malignancy was significantly higher than benign lesion. BIRADS in combination with stiff rim sign can have a satisfactory diagnostic performance.

\section{Abbreviations}

AUC: area under the curve; BI-RADS: Breast Imaging and Reporting and Data Systems; CNB: core needle biopsy; DCIS: ductal carcinoma in situ; IDC: invasive ductal carcinoma; ROC: receiver operating 
characteristic; SE: Strain elastography; SWE: Shear wave elastography; VTIQ: virtual touch tissue imaging quantification

\section{Declarations}

\section{Acknowledgements}

Thank all the doctors of Drumtower Hospital affiliated to Medical College of Nanjing University

\section{Authors' contributions}

Kong WT organized the data and wrote the paper. Wang Y, Zhou WJ, Zhang YD searched and analysed the data. Zhuang XM collected the clinical data. Wang WP and Wu M revised the paper. All authors read and approved the manuscript.

\section{Funding}

This work was supported by the Nanjing Medical Science and Technique Development Foundation (QRX17011). Part of the expense for contrast agent was funded by this project.

\section{Availability of data and materials}

Please contact the author for further data.

\section{Ethics approval and consent to participate}

This study was approved by Drumtower Hospital Ethics committee of Nanjing University. The study had adhered to the law of China and the 2008 Helsinki Declaration. Signed written informed consent was obtained from all participants.

\section{Consent for publication}

Not applicable.

\section{Competing interests}

The authors declare that they have no conflicts of interest. 


\section{Author details}

${ }_{1}$ Department of Ultrasound, Drumtower Hospital, Medical College of Nanjing University, Nanjing 210003, China. ${ }_{2}$ Department of Thyroid and Breast Surgery, Drumtower Hospital, Medical College of Nanjing University, Nanjing 210003, China

\section{References}

Barr RG, Nakashima K, Amy D, Cosgrove D, Farrokh A, Schafer F, et al. WFUMB guidelines and recommendations for clinical use of ultrasound elastography: Part 2: breast. Ultrasound Med Biol. 2015; 41: 1148-60.

Youk JH, Son EJ, Gweon HM, Kim H, Park YJ, Kim JA. Comparison of strain and shear wave elastography for the differentiation of benign from malignant breast lesions, combined with B-mode ultrasonography: qualitative and quantitative assessments. Ultrasound Med Biol. 2014; 40: 2336-44.

Sigrist RMS, Liau J, Kaffas AE, Chammas MC, Willmann JK. Ultrasound Elastography: Review of Techniques and Clinical Applications. Theranostics. 2017; 7: 1303-29.

Liu B, Zheng Y, Huang G, Lin M, Shan Q, Lu Y, et al. Breast Lesions: Quantitative Diagnosis Using Ultrasound Shear Wave Elastography-A Systematic Review and Meta--Analysis. Ultrasound Med Biol. 2016; 42: 835-47.

Hari S, Paul SB, Vidyasagar R, Dhamija E, Adarsh AD, Thulkar S, et al. Breast mass characterization using shear wave elastography and ultrasound. Diagn Interv Imaging. 2018; 99: 699-707.

Song EJ, Sohn YM, Seo M. Tumor stiffness measured by quantitative and qualitative shear wave elastography of breast cancer. Br J Radiol. 2018; 91: 20170830.

Zhou J, Zhan W, Dong Y, Yang Z, Zhou C. Stiffness of the surrounding tissue of breast lesions evaluated by ultrasound elastography. Eur Radiol. 2014; 24: 1659-67.

Park HS, Shin HJ, Shin KC, Cha JH, Chae EY, Choi WJ, et al. Comparison of peritumoral stromal tissue stiffness obtained by shear waveelastography between benign and malignant breast lesions. Acta Radiol. 2018; 59: 1168-75.

A. Evans, P. Whelehan, K. Thomson, D. McLean, K. Brauer, C. Purdie, et al. ThompsonInvasive breast cancer: relationship between shear-wave elastographic findings and histologic prognostic factors. Radiology. 2012; 263: 673-7.

American College of Radiology. Breast Imaging Reporting and Data System(BI-RADS) Mammography. 5th ed. American College of Radiology, Reston, Va; 2013. 
Luo J, Cao Y, Nian W, Zeng X, Zhang H, Yue Y, et al. Benefit of Shear-wave Elastography in the differential diagnosis of breast lesion: a diagnostic meta-analysis. Med Ultrason. 2018; 1: 43-9.

Li XL, Xu HX, Bo XW, Liu BJ, Huang X, Li DD, et al. Value of Virtual Touch Tissue Imaging Quantification for Evaluation of Ultrasound Breast Imaging-Reporting and Data System Category 4 Lesions. Ultrasound Med Biol. 2016; 42: 2050-7.

Liu Y, Huang Y, Han J, Wang J, Li F, Zhou J. Association Between Shear Wave Elastography of Virtual Touch Tissue Imaging Quantification Parameters and the Ki-67 Proliferation Status in Luminal-Type Breast Cancer. J Ultrasound Med. 2019; 38: 73-80.

Xiao Y, Yu Y, Niu L, Qian M, Deng Z, Qiu W, et al. Quantitative evaluation of peripheral tissue elasticity for ultrasound-detected breast lesions. Clin Radiol. 2016; 71: 896-904.

Wang ZL, Sun L, Li Y, Li N. Relationship between elasticity and collagen fiber content in breast disease: a preliminary report. Ultrasonics. 2015; 57: 44-9.

Evans A, Whelehan P, Thomson K, McLean D, Brauer K, Purdie C, et al. Quantitative shear wave ultrasound elastography: initial experience in solid breast masses. Breast Cancer Res. 2010; 12: R104.

Kapetas P, Pinker-Domenig K, Woitek R, Clauser P, Bernathova M, Spick C, et al. Clinical application of Acoustic Radiation Force Impulse Imaging with Virtual Touch IQ in breast ultrasound: diagnostic performance and reproducibility of a new technique. Acta Radiol. 2017; 58: 140-7.

Vinnicomber SJ, Whelehan P, Thomson K, Mclean D, Purdie CA, Jordan LB, et al. What are the characteristics of breast cancers misclassified as benign by quantitative ultrasound shear wave elastography? Eur Radiol. 2014; 24: 921-6.

Chen YP, Han T, Wu R, Yao MH, Xu G, Zhao LX, et al. Comparison of Virtual Touch Tissue Quantification and Virtual Touch Tissue Imaging Quantification for diagnosis of solid breast tumors of different sizes. Clin Hemorheol Microcirc. 2016; 64: 235-44.

\section{Figures}




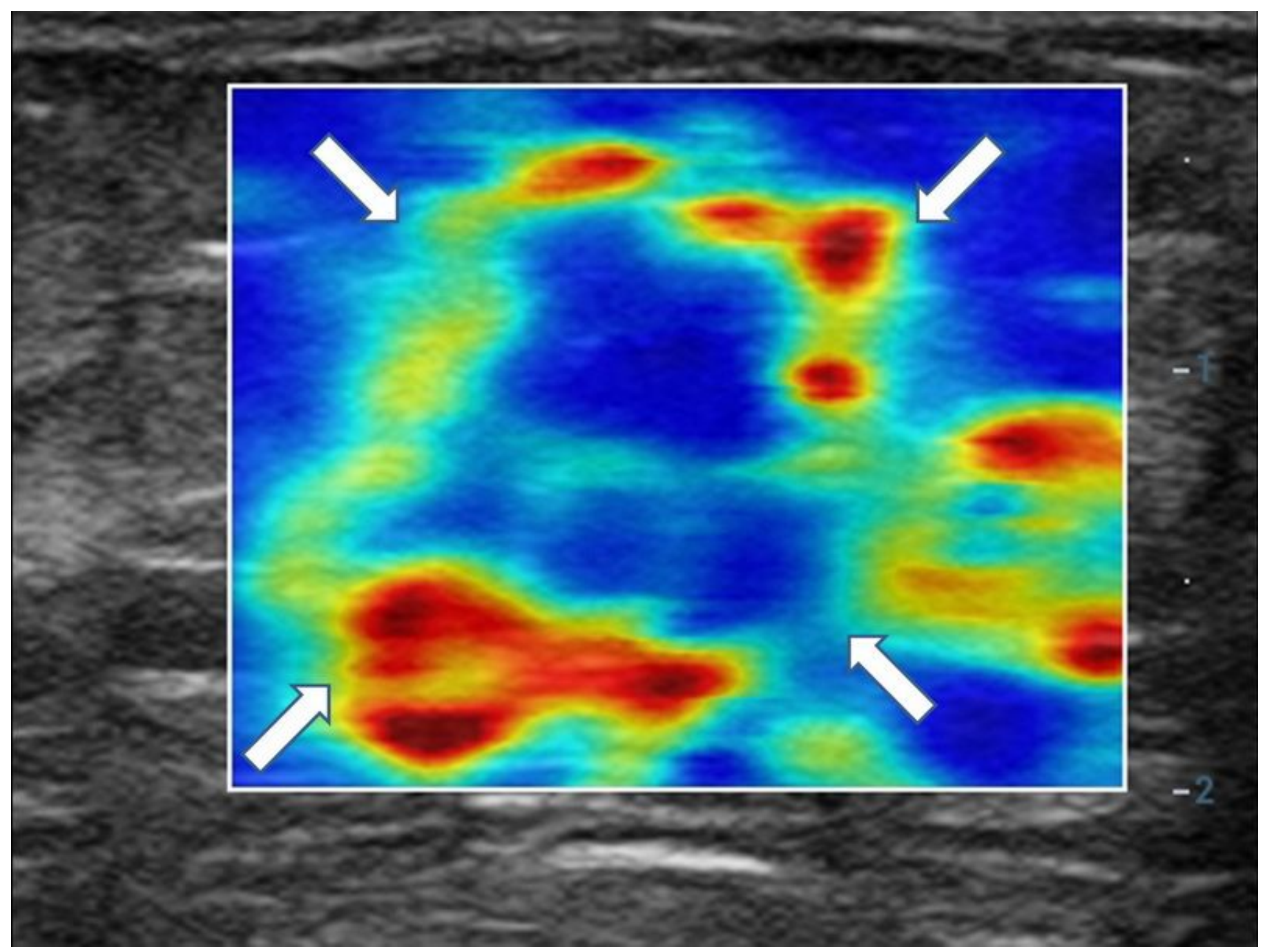

\section{Figure 1}

The stiff rim sign. The breast lesion was surrounded by red and orange halo, which may be caused by desmoplastic reaction. 


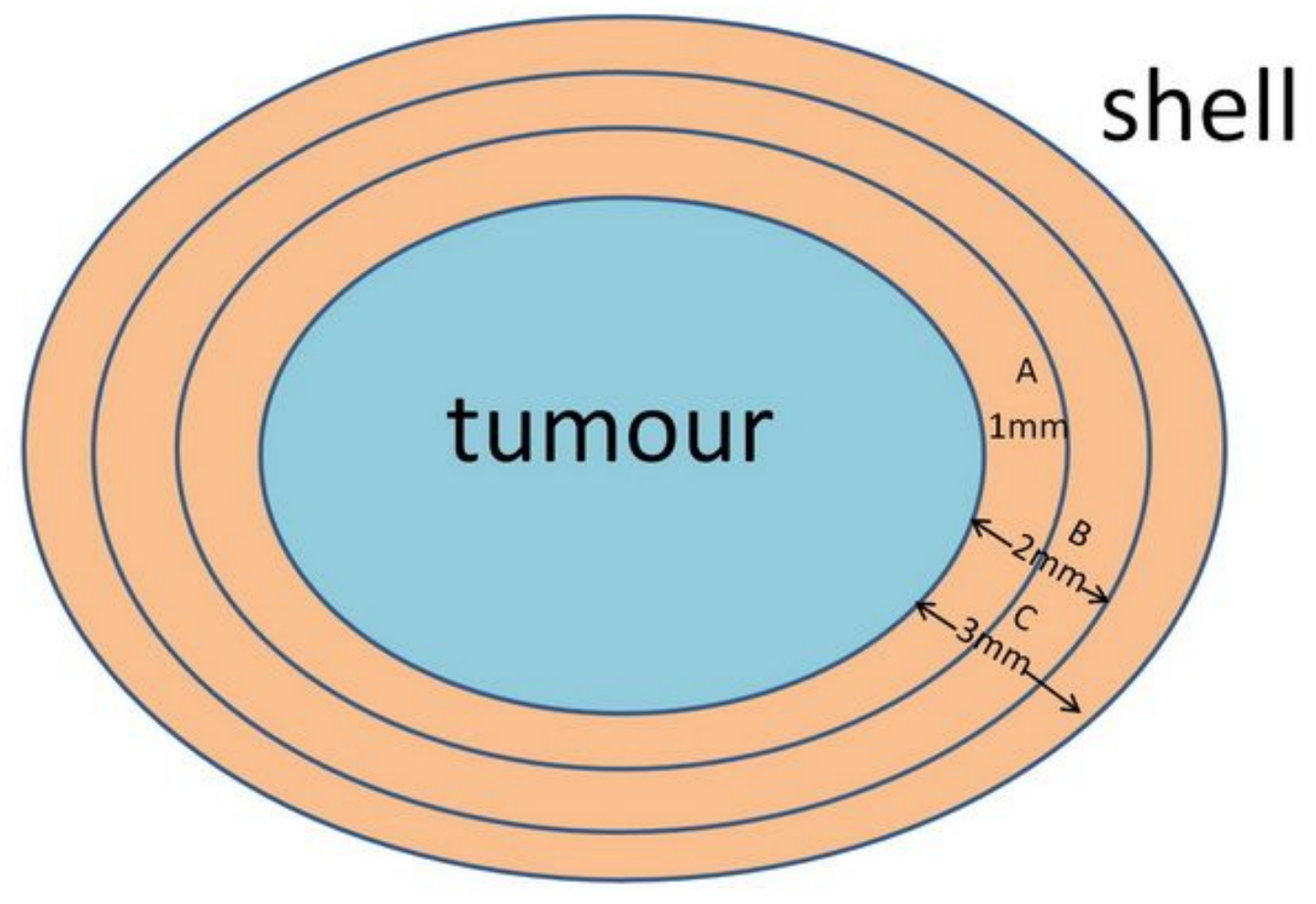

Figure 2

The sketch map of the method concerning quantitative measurement of shell. After drawing the outline of breast lesion manually, we drew the shell around lesion, with width of $1 \mathrm{~mm}$ (shell A), $2 \mathrm{~mm}$ (shell B), and $3 \mathrm{~mm}$ (shell C), respectively. 


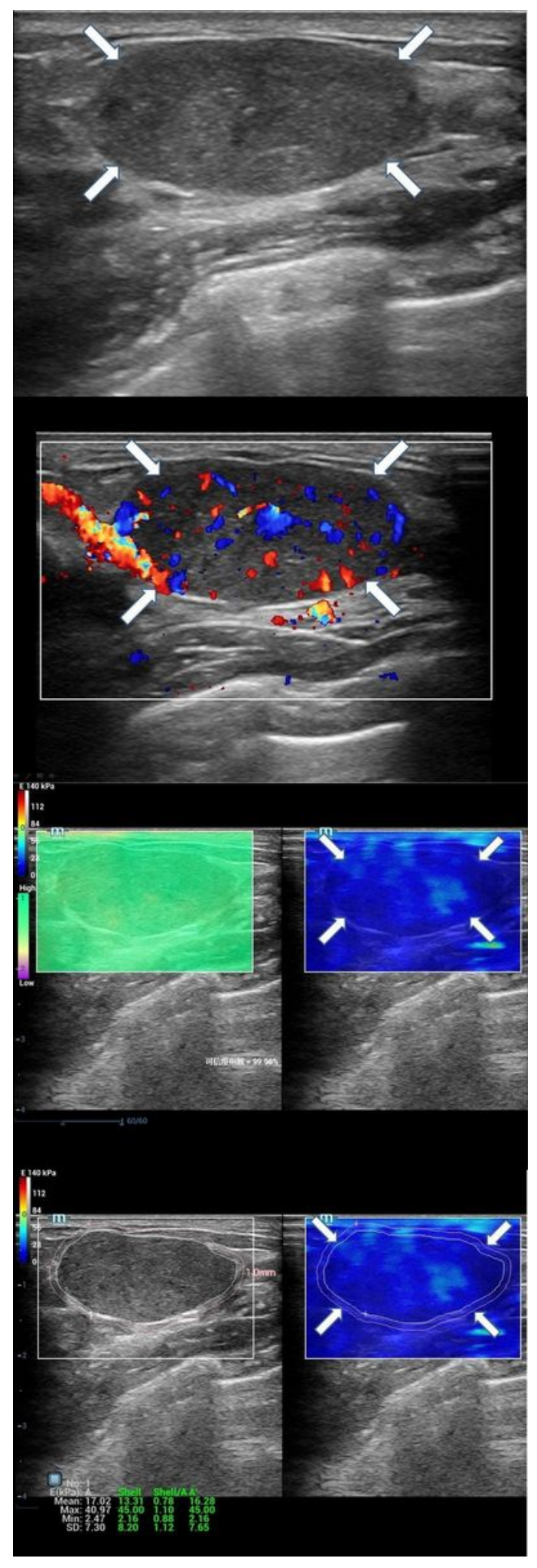

\section{Figure 3}

A fibroadenoma in a 24-year-old woman. A regular, hypo-echogenic solid breast lesion was seen on Bmode US (a), which was classified as BI-RADS 3. CDFI showed abundant blood flow signal in the lesion (b). The lesion was soft represented as blue (c, right) with a satisfactory quality of elastographic image (c, left). Quantitative measurement showed the Emax, Emean of shell and shell/lesion ratio were 45.0 $\mathrm{Kpa}, 13.3 \mathrm{Kpa}$ and 0.78 (d). 

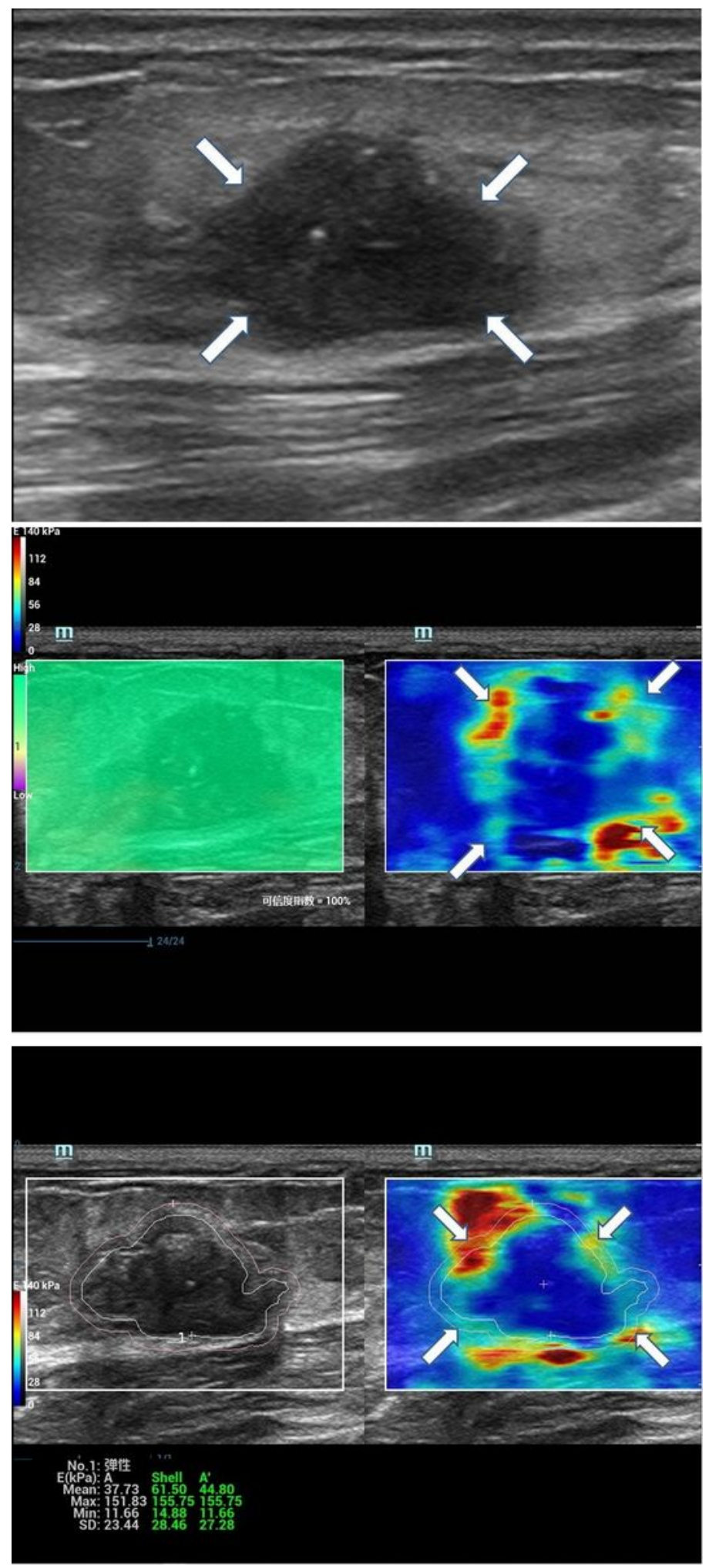

\section{Figure 4}

A invasive ductal carcinoma in a 47-year woman. B-mode US showed an irregular and heterogeneous lesion with micro-calcification (a). There was a stiff rim sign in SWE mode (b). The Emax, Emean of shell and shell/lesion ratio were $155.8 \mathrm{Kpa}, 61.5 \mathrm{Kpa}$ and 1.63 (c). 


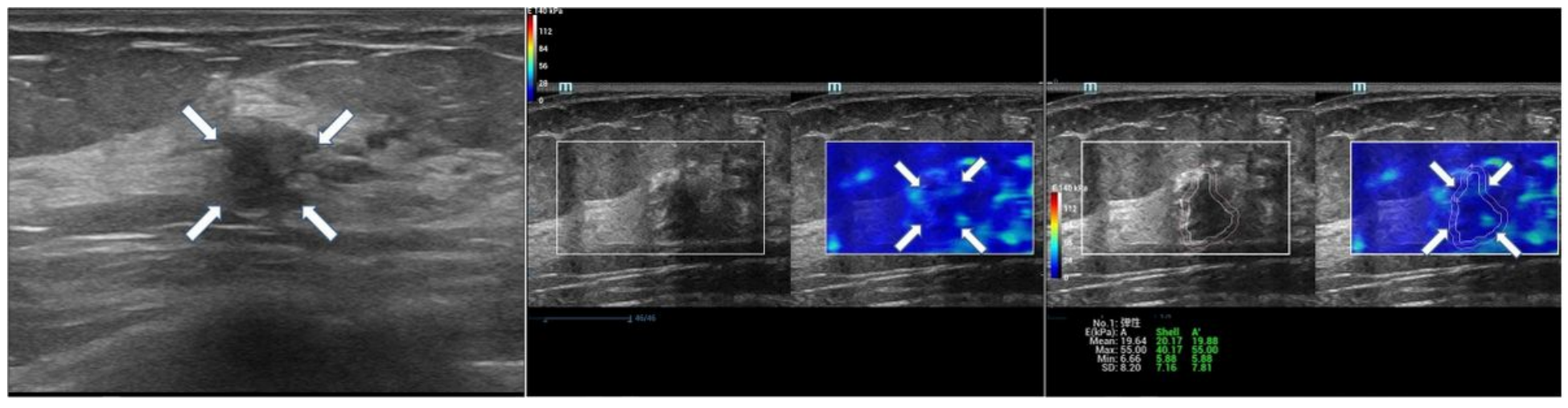

\section{Figure 5}

A invasive ductal carcinoma in a 55-year woman. On B-mode US, the small breast lesion $(9 \mathrm{~mm})$ showed malignant characteristics, which can be classified as BI-RADS-4c (a). However, the lesion was soft showing almost blue on SWE mode (b), with low Emax, Emean of shell and shell/lesion ratio of $40.2 \mathrm{Kpa}$, $20.2 \mathrm{Kpa}$ and 1.03 , respectively.

\section{Supplementary Files}

This is a list of supplementary files associated with this preprint. Click to download.

- table.docx 